\title{
Optimization of C5 separation and production process
}

\author{
Yonghong Wang \\ Department of automatic control, Nanjing College of Chemical Technology, Nanjing, 210048, China
}

\section{Keywords: C5; separation; optimization}

\begin{abstract}
C5 separation according to actual conditions of C5 production, studies the process of each separation unit, optimizes process conditions of each separation unit and analyzes effects of each device and factor on the whole process. Chinese and foreign research background, theoretical model and process optimization of C5 separation process are mainly analyzed to confirm the optimization scheme and requirements.

Plentiful by-product - C5 fraction will produce in the process of producing ethylene with petroleum hydrocarbon through pyrolysis. The output of C5 fraction is $10 \%-20 \%$ of that of ethylene. C5 fraction contains over 30 multiple components with the similar boiling point, including isoprene, cyclopentadiene and pentadiene. The content of the three is high, accounting for $40 \%-50 \%$. Meanwhile, the three substances are fundamental raw materials used to produce resin, rubber, spice, medicine and other fine chemical products. Fraction output of C5 as a by-product of ethylene also grows rapidly. Since the substance used to split C5 fraction consist of more than 20 components with very similar boiling point which can easily form the azeotrope. It is difficult to isolate qualified C5 diolefin. The technology of the whole process is complex. How to develop further processing in a better and faster manner, improve multipurpose utilization rate of C5 fraction, reduce energy consumption in C5 separation process, improve product performance and boost the added value and optimize C5 separation process is very significant.
\end{abstract}

\section{Progress of C5 separation}

The fraction used to split C5 contains multiple components with good utilization value. If they are directly applied, economic benefit is poor. So, more proper separation method is needed to separate components. The process of splitting C5 fraction is the process of separating and recycling cyclopentadiene, isoprene and pentadiene with high utilization value.

There are many components used to split C5 fraction, and the boiling point of each component approaches. Besides, each component can generate azeotropy. Thus, it is hard for common distillation methods to separate C5 fraction. Currently, dicyclopentadiene is first separated through heating dimerization in industries, and then solvent extraction and distillation are adopted to separate pentadiene and isoprene.

Reactive distillation technology is also adopted to separate and split C5 fraction diolefin. After C5 fraction is sent to reactive distillation tower, pentadiene C5 hydrocarbon at the top of the tower will be sent to extraction distillation tower, while dicyclopentadiene and other heavy components in the kettle of the tower will be sent to the de-heavy tower. When materials at the top of the de-heavy tower are sent to pentadiene tower, C5 hydrocarbon is at the top of the tower. When materials at the kettle of de-heavy tower are sent to dicyclopentadiene tower, C6 and other hydrocarbons with light boiling point will be removed at the top of the tower, while dicyclopentadiene is extracted from vapor phase at the kettle of the tower. Thus, when dimerization reaction happens to cyclopentadiene, isoprene is separated out of the top of the tower, while C5 fraction is distilled into dicyclopentadiene and separates pentadiene and isoprene.

\section{Theoretical model and its optimization for C5 separation}

Thermodynamic model for C5 separation can be classified into two categories: activity 
coefficient method and state equation method. In accordance with mixed C5 equilibrium data in actual production, accurate and reliable thermodynamic model is set up. This is vital for accuracy of mixed C5 separation process.

\subsection{Happel model for C5 extractive distillation}

Happel model for C5 extractive distillation develops on the basis of Margule model. This model combines theories and experience. So, its scope of application is wider than pure empirical models, and it is more accurate than pure empirical models. Among the models used to describe C5 extractive distillation, the formula of Happel model is the simplest, and Happel model is the most convenient during mathematical treatment. it is table to calculate the distillation tower with matrix method in the principle of automatic control. It is more liable to convergence. However, this model has a defect in describing temperature variation regularity. This arises from simplification of this model. If complete Wilson model is adopted in actual process, this defect can be overcome. It can be used in full concentration range of the solvent. The application model of vapor-liquid equilibrium in C4 extractive distillation process is applied in C5 extractive distillation system. Such method utilizes end value constant to correlate component activity coefficient. If component concentration change range is large, larger errors will occur. Especially in the de-solvent section of extraction distillation tower, relevebt solvent concentration approaches 0 . Thus, such method often makes iterative computations of bubble point fail.

2.2 Comparison of new process and conventional process

The boiling point of components used to split C5 fraction approaches, and two-component and three-component are generated. So, common distillation methods cannot be used to separate and gain products with high purity. Extractive distillation is used to reach the purpose of separating high-purity isoprene.

The operation rules of extractive distillation tower are gained through analyzing pressure and temperature of extractive distillation tower as well as vapor - liquid phase flow rate distribution. Through further investigating effects of major parameter theoretical plate, ratio of solvent and feed entrance point on thermal load and separation effects, the optimal operation process and conditions are finally confirmed to reduce energy consumption and reduce solvent loss.

Conventional process can only produce about 80\%DCPD. This indicates thermal dimerization has certain defects before CPD and IP are separated. This not just affects mass concentration of DCPD, but increases consumption of CPD and IP due to codimerization. From the dynamics perspective of dimerization reaction, PD activity is much lower than IP activity. So, dimerization of CPD and IP should be milder and be neglected. Therefore, conventional C5 separation process is summarized as follows: (1) all PD and IP participate in reactions in dimerization reactor so that IP seriously loses. Meanwhile, DCPD product quality is affected; (2) the design and operation conditions of de-heavy tower are relatively rigorous. IP loss tower and scale formation at tower bottom may be caused; (3) the volume of dipolymer reactor is huge, so it is hard to control and design it; (4) the temperature at the bottom of pre-deheavy tower is quite high and scale often forms.

The improved process organically integrates IP separation tower and CPD recovery tower through return cable setting from the bottom of CPD recovery tower to IP separation tower to jointly fulfill separation of CPD and IP. In the whole process, stripping section of CPD recovery tower and rectifying section of IP separation tower will reduce. Besides, in combination of control requirements of the two towers, design difficulty of the two towers will lower.

\section{Optimization of C5 separation process}

Major products of mixed C5 separation include pentadiene, polymer-grade isoprene and dicyclopentadiene. Based on features and natures of these separation objects, conventional rectification, dimerization reaction, decompression rectification and extractive distillation are adopted. The most outstanding feature of C5 separating unit is that C5 fraction can easily form autopolymer. So, during selecting detection instruments, the damage of autopolymer formed in the production process on each detection instrument must be considered to prevent distortion of the 
detected value.

3.1 Optimization of C5 preprocessing unit

The function of preprocessing unit is to remove most hydrocarbon blocks in C5 fraction. Most cyclopentadiene is dimerized into dicyclopentadiene through thermal dimerization. Then, dicyclopentadiene is separated to lighten the burden of component separation in the following process. In order to separate more isoprene, cyclopentadiene and other valuable components, isoprene removal tower is added between de-light tower and dipolymer reactor during optimization of preprocessing unit. The functions of isoprene removal tower are as follows: collect fraction rich in isoprene at the top of the tower and then enter isoprene rectification unit for separation; tower bottoms enter dipolymer reactor. The application of isoprene removal tower greatly reduces the content of isoprene in dimerization reaction and effectively increases dicyclopentadiene in dimerization reactants, while the content of the by-product isopropenyl norbornene declines significantly. Multipurpose utilization rate of C5 fraction improves.

3.2 Optimization of the first extractive distillation unit

The first extractive distillation unit is extractive distillation of decyclized C5 after pretreatment. The main devices of this link include the first extractive distillation tower, de-heavy tower and stripping tower. Extractive distillation is applicable to separating the solutions whose components have similar volatility. To improve vapor-liquid equilibrium relationship among components separated, proper separation medium is added. The separation medium reacts with different components so that interaction force among each component weakens. Thus, the components with similar volatility are more liable to rectify and separate. The extractive distillation tower is a multi-feed and multi-component complex tower. Tower temperature and vapor-liquid phase flow rate change greatly. To reduce solvent loss, improve multipurpose utilization rate of C5 fraction and reduce energy consumption, optimal operating conditions of extractive distillation tower are needed. Therefore, rational charging heat condition needs selecting to make temperature distribution of extractive distillation tower tend to be normal. Secondly, plate number imposes impacts on reflux ratio. Thirdly, ratio of solvent is also one of significant factors influencing separation effect. Finally, feed entrance point needs optimization.

3.3 Optimization of the second extractive distillation unit

The second extractive distillation unit caries out secondary extractive distillation of chemical-grade isoprene gained from the first extractive distillation unit to remove cyclopentadiene and hydrocarbon blocks and finally gain polymer-grade isoprene. The devices involved in this process mainly include the second extractive distillation tower, de-light tower and stripping tower. Similar to the first extractive distillation unit, optimization of this link also starts with the operation. The optimal operating coefficients are confirmed through analyzing reflux ratio, theoretical plate number, ratio of solvent and feed entrance point so as to gain the largest separation effect. It is necessary to analyze operating coefficients in C5 extractive distillation process so as to optimize operating coefficients and relevant equipment parameters.

3.4 Optimization of solvent recovery unit

Solvent recovery unit involves poor solvent from the first extractive distillation unit, solvent underground tank and solvent recuperation kettle. Pressure reduction and distillation are conducted for solvent recuperation kettle. The purpose of heating residues is fulfilled through heating U-type tube bundle in the kettle. In solvent recovery unit, heavy black residues and colloid will occur during heating solvent recuperation kettle. Accumulation of these substances makes it hard to continue to evaporate the solvent, thus influencing changeover of recuperation kettle. Moreover, in slagging process, empyreumatic oil cannot be discharged smoothly and thoroughly. Some will adhere to heat exchange tube. High-pressure hydraulic giant cannot be cleaned with water. Manual cleaning is needed. This goes against improvement of service life of recuperation kettle. Optimization of production flow of solvent recovery unit is as follows: regenerated solvent first enters the reboiler for heating and then enters flashing tank. Solvent vapor comes out from the top, and liquid material flow is discharged from the bottom. Liquid material flow brand is discharged from the bottom. Some is sent to circulating solvent feed pipeline, and some enters waste residue 
tank. If liquid temperature in the flashing tank continuously rises, this indicates empyreumatic oil accumulates in quantity. At this moment, it is necessary to open the valve from the pump to the waste residue tank. The valve will not be opened until liquid temperature in the flashing tank drops to the normal value. Vapor-state material flow at the top of flashing tank through the condenser is sent to solvent refining tower by a pump. Then, rectification is conducted. Water, C5 hydrocarbon and C5 low polymer appear at the top of the tower. The solvent with the purity exceeding $99.5 \%$ is gained at the kettle of the tower and returns to the extraction unit to supplement solvent, which greatly improves resource utilization rate.

Optimized solvent recovery unit separates the heater and recuperation kettle. A solvent circulating pump is added to reduce heat exchange area and reduce investment. The whole production process fulfils airtight continuous production, which reduces the possibility of polymer exposed in the air and reduces pollutant discharge. The circulating pump added has stirring function and enhances material liquidity in the flashing tank, promotes separation of solvent and empyreumatic oil and lighten operation difficulties.

\section{Optimization of self-control scheme}

Optimization of self-control scheme is very vital during separating mixed C5. The following aspects are involved.

(1) The material C5 is charged through dipolymer reactor. Meanwhile, flow liquid level is measured. If liquid level reaches the set value, self-control regulating valve needs to be closed, and circulating regulating valve opens.

(2) Vapor-phase discharge flow is regulated in series to control feed quantity control of C4 tower. It is a two-circuit regulating system in which main and minor regulators are connected in series. Main regulator outputs regulatory signals according to the given value and deviations of main parameters to serve as the given value of minor regulator. Meanwhile, the minor regulator receives the given value and signals of minor parameters and controls the regulating mechanism. This improves dynamic feature of regulation objects, and the work efficiency of regulating system improves greatly. Besides, real-time adaptation ability and anti-disturbance ability of the whole system boosts.

(3) Non-contact sensor is used to detect liquid level change in the container in an timely and accurate manner, send out control signals and alarming signals, and prevent liquid overflow and machine operation without water in actual applications.

(4) PID control and fuzzy control are combined to control C5 separation liquid level. Fuzzy control decides the proportion, integral and differential of PID control to better adapt conditions and parameter changes of C5 separation liquid level control system.

\section{Conclusions}

As ethylene industry and derivative industry develop and requirements for energy conservation, emission reduction and environmental protection increase, during C5 separation, it is required to actively study new technology, optimize exiting techniques, solve existing problems, boost multipurpose utilization rate of C5 fraction and reduce separation energy consumption. Moreover, it is required to carry out simulation analysis of C5 separation process, add or decrease relevant equipment. Optimize production process and equipment operation parameters, make sure C5 separation is conduct smoothly, reduce energy consumption and production investment and improve recover rate and quality of isoprene.

\section{References}

[1] Sun Chao, Yao Benzhen, Fu Jiansong et al., Optimization of preprocessing unit process of c5 seperation [J]. Petrochemical Engineering, 2013, 42(8):880-885. 
[2] Qi Shujing, Wang Feihu, Zhao Xiaowei et al., Progress of Chinese C5 separation technology [J]. Elastomer, 2009,19(5):71-75.

[3 Sha Yu, Ge Chunfang, Development and research of advanced C5 separation technology [J]. Petroleum Refinery Engineering, 2010, 40(7):5-8.

[4] Wang Chunlei, Wang Yu, Qu Yun et al., Study on crude C5 separation technology [J]. Chemical Intermediate, 2012, 09(4):53-56,48.

[5] Ma Liguo, Improvement and optimization of solvent recovery system process of C5 separation device with DMF [J]. Ethylene Industry, 2014,(2):43-46.

[6] Fan Yu, [J]. Modern Chemical Industry, 1995(4):3-32.

[7] Wu Junsheng, Shao Huihe, Rectification design, operation and control [M]. Beijing: China Petrochemical Press, 1997.12

[8] Zeng Aiwu, Application of rectification technology in fine chemical separation [J]. Fine Chemical Raw Materials and Intermediates, 2005, (9): 8-11 